\title{
False-positive ELISA Reactivity to Hepatitis B Virus Surface Antigen (HBsAg)
}

It is claimed that the possibility of non-specific reactions in ELISA tests for HBsAg has been substantially eliminated (1), though like any other highly sensitive immune test system, ELISA does still have the potential of yielding false-positive reactions. Such reactivity in the hepatitis $B$ virus (HBV) hyperendemic focus of the Gizan Area of Saudi Arabia was encountered using a two-titer test system, involving a full $\mathrm{HBV}$ serological profile and a neutralization test.

During the period September 1984 to December 1985, 1512 sera were drawn from Saudis either during a voluntary blood donation or while seeking treatment for minor ailments at the Gizan General Hospital in the Gizan Area (2). The samples were tested initially using Abbott ELISA kits (ABBOTT Laboratories, North Chicago, Il.) for HBsAg (Auszyme ${ }^{\otimes}$-II), antibody to HBV surface antigen (anti-HBs, Ausab-EIA) and antibody to HBV core antigen (anti-HBc, Corzyme TM). Of the 1512 sera, 210 were positive for $\mathrm{HBsAg}$ and anti-HBc, while 17 were positive for anti-HBs as well for both $\mathrm{HBsAg}$ and anti-HBc. Six sera, examined during three re-tests, were found positive only for $\mathrm{HBsAg}$. They were tested for $\mathrm{HBeAg}$, anti$\mathrm{HBe}$ and $\mathrm{IgM}$ antibody for $\mathrm{HBV}$ core antigen. Two were positive for $\mathrm{HBeAg}$ and $\mathrm{IgM}$ anti-HBc but negative for anti-HBe. These two could not be re-tested for $\mathrm{HBsAg}$ quantification since they had been exhausted during the above tests. They were labelled as being in the late incubation period, while the remaining four sera were negative. In the remaining four specimens, a neutralization test (NT) by overnight incubation at room temperature, was carried out on their $0.2 \mathrm{ml}$ volumes with $0.2 \mathrm{ml}$ of either a serum negative for anti-HBs or an Abbott anti-HBs preparation containing $100 \mathrm{mIU} / \mathrm{ml}$. As a control for the NT, known HBsAg-positive and negative specimens were set up in parallel. ELISA reactivity was tested in each mixture on the following morning.
ELISA reactivity in four specimens remained unaffected by the anti-HBs-negative or positive sera. No further specimen aliquots were available for a comparison of the results with the Abbott Auszyme II confirmatory neutralization test kit. All four specimens were labelled negative for serological evidence of exposure to $\mathrm{HBV}$.

In HBV hyperendemic areas, simultaneous testing of all serum samples for $\mathrm{HBs} \mathrm{Ag}$, anti-HBs and anti-HBc should confirm any dubious ELISA reactivity for HBsAg in any specimen. Exclusively HBsAg-positive specimens, if also positive for the $\mathrm{HBeAg}$ and anti-HBc IgM class, can be categorised as belonging to the late incubation period. A back-up neutralization test, as demonstrated by four HBsAg-negative Saudis in our series, should eliminate false alarms of $\mathrm{HBV}$ antigenemia in otherwise still unexposed persons. Pending in-depth evaluation of the nature of non-specific HBsAg reactors in ELISA tests, the possibility of leaving any $\mathrm{HBV}$-susceptible person, unimmunized on the basis of false-positive ELISA reactivity, as the four in our series, should be minimized in any HBV hyperendemic area.

S. C. Arya, C. M. Parande, S. J. Ashraf

\section{Literature}

1. Abbott Laboratories Diagnostic Division: Auszyme II. Antibody to hepatitis B surface antigen (goat): Peroxidase (horseradish) conjugate. May 1985, p. 13.

2. Arya, S. C., Ashraf, S. J., Parande, C. M., El-Sayed, M., Sahay, R., Ageel, A. R., Tobeiqi, M. S.: Hepatitis B virus in Gizan, Saudi Arabia. J. Med. Virol. 17 (1985) 267-274.

Dr. S. C. Arya, Dr. S. J. Ashraf, King Fahad Central Hospital, Department of Medicine, P.O. Box 204, Gizan, Saudi Arabia; Dr. C. M. Parande, Gizan General Hospital, Gizan, Saudi Arabia. 